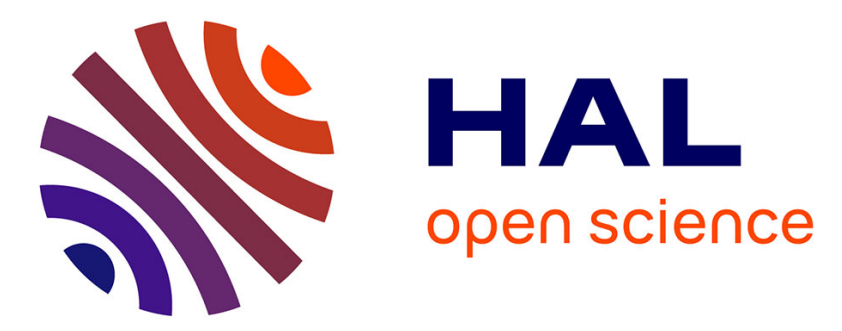

\title{
Effective quality control of incoming potatoes as an acrylamide mitigation strategy for the French fries industry
}

Raquel C. Medeiros Vinci, Frédéric Mestdagh, Nathalie de Muer, Carlos van Peteghem, Bruno de Meulenaer

\section{To cite this version:}

Raquel C. Medeiros Vinci, Frédéric Mestdagh, Nathalie de Muer, Carlos van Peteghem, Bruno de Meulenaer. Effective quality control of incoming potatoes as an acrylamide mitigation strategy for the French fries industry. Food Additives and Contaminants, 2010, 27 (04), pp.417-425. 10.1080/19440040903453276 . hal-00576996

\section{HAL Id: hal-00576996 https://hal.science/hal-00576996}

Submitted on 16 Mar 2011

HAL is a multi-disciplinary open access archive for the deposit and dissemination of scientific research documents, whether they are published or not. The documents may come from teaching and research institutions in France or abroad, or from public or private research centers.
L'archive ouverte pluridisciplinaire HAL, est destinée au dépôt et à la diffusion de documents scientifiques de niveau recherche, publiés ou non, émanant des établissements d'enseignement et de recherche français ou étrangers, des laboratoires publics ou privés. 


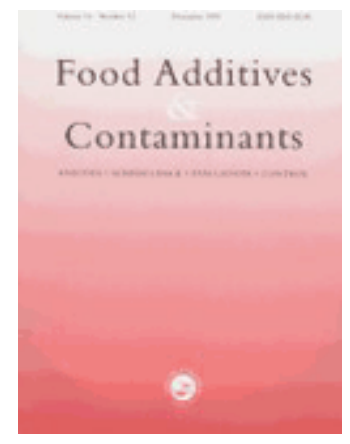

\section{Effective quality control of incoming potatoes as an acrylamide mitigation strategy for the French fries industry}

\begin{tabular}{|r|l|}
\hline Journal: & Food Additives and Contaminants \\
\hline Manuscript ID: & TFAC-2009-254.R1 \\
\hline Manuscript Type: & Original Research Paper \\
\hline Author: & 25 -Oct-2009 \\
\hline Complete List of Authors: & $\begin{array}{l}\text { C. Medeiros Vinci, Raquel; Ghent University, Department of Food } \\
\text { Safety and Food Quality } \\
\text { Mestdagh, Frédéric; Ghent University, Department of Food Safety } \\
\text { and Food Quality } \\
\text { De Muer, Nathalie; Ghent University, Department of Food Safety } \\
\text { and Food Quality } \\
\text { Van Peteghem, Carlos; Ghent university, Department of Bioanalysis } \\
\text { De Meulenaer, Bruno; Ghent University, Department of Food Safety } \\
\text { and Food Quality }\end{array}$ \\
\hline Methods/Techniques: & Regression, Chromatography - LC/MS \\
\hline Additives/Contaminants: & Acrylamide \\
\hline Food Types: & Potatoes \\
\hline & \\
\hline
\end{tabular}

\section{SCHOLARONE"} Manuscripts 


\section{Effective quality control of incoming potatoes as an acrylamide}

2 mitigation strategy for the French fries industry

4 Raquel Medeiros Vinc $i^{a}$, Frédéric Mestdagh ${ }^{a}$, Nathalie De Mue $r^{a}$, Carlos Van Peteghem ${ }^{b}$, Bruno De 5 Meulenaer $^{a}$

6

$7 \quad{ }^{a}$ NutriFOODchem Unit, Department of Food Safety and Food Quality, Faculty of Bioscience

8 Engineering, Ghent University, Coupure Links 653, B-9000 Ghent, Belgium, ${ }^{\mathrm{b}}$ Department of

9 Bioanalysis, Faculty of Pharmaceutical Sciences, Ghent University, Harelbekestraat 72, B-9000 Ghent, 10 Belgium

16 * Corresponding author. Email address: Bruno.DeMeulenaer@UGent.be 
17 Abstract

18 The correlation between sugar levels in raw material (potatoes), brown colouring and formation of 19 acrylamide in French fries was investigated. Such a study would allow the identification of incoming 20 potatoes (raw material) with high potential for acrylamide-formation. Ten different potato varieties 21 commonly used in the Western European French fries industry were stored at $8^{\circ} \mathrm{C}$ and samples were 22 taken throughout the storage time. The current quality control used in the French fries industry for 23 incoming potatoes is poorly correlated with acrylamide in the final product $(\mathrm{R}=0.74)$. Changing the 24 quality control parameter from colour to reducing sugars in raw material did not improve the correlation $25(\mathrm{R}=0.72)$. The best correlation was obtained with Agtron colour measurement after blanching and a 26 two stage frying $(\mathrm{R}=-0.88)$. From this study it could be concluded that alternative entrance control 27 measurements could provide a better mitigation of the acrylamide issue in French fries from the start of 28 the production. These alternatives however are less cost-effective and more difficult to implement.

29 KEYWORDS: Acrylamide; Quality control; Colour; Reducing sugars; French fries 


\section{$30 \quad$ Introduction}

31 Fried potato products are very familiar and popular among many gastronomic cultures. French fries 32 (also known as chips or fries), either fresh or pre-frozen, are widely industrially produced and consumed 33 after a final deep fat frying step. During this process, acrylamide, a probable human carcinogen is 34 formed (IARC 1994). Exposure studies demonstrate that French fries represent a great source of dietary 35 acrylamide for consumers (Mestdagh et al. 2007).

36 This process contaminant is formed during the heating process at temperatures above $120^{\circ} \mathrm{C}(\mathrm{Tareke}$ 37 et al. 2002) and is known to be linked to the Maillard browning reaction. The free amino acid asparagine 38 and the reducing sugars (fructose and glucose) have been identified as the main precursors for its 39 formation (Becalski et al. 2003; Mottram et al. 2002; Stadler et al. 2002). Since generally the amount of free asparagine in potato tubers largely exceeds the amount of reducing sugars, it is the latter which determines the degree of acrylamide formation and the Maillard browning upon frying (Amrein et al. 2003; De Wilde et al. 2005). Multiple factors have been demonstrated to influence the reducing sugar content in potatoes, for example, potato cultivar (Biedermann et al. 2002; De Wilde et al. 2006), storage time and temperature (Blenkinsop et al. 2002; De Wilde et al. 2005).

For the mitigation of acrylamide formation in French fries, several strategies may be considered, for instance, the selection and storage of the raw material, adaptation of processing conditions, and pretreatments applied prior to par-frying (CIAA 2006; Foot et al. 2007). Predicting the sensitivity of incoming potatoes for the formation of acrylamide would provide a quality control tool of great aid for the French fry industry in their food safety management system. This would allow preventing the 50 problem at the start of the production process. The current quality control at the reception point of the 51 raw material potatoes is done based on colour evaluation with USDA/Munsell colour chart (after a short 52 frying test, typically $180^{\circ} \mathrm{C}$ for $3 \mathrm{~min}$ ) (UEITP 2007). This quality control relates raw material to colour 53 specifications of final product (customers demand) and accordingly, either raw material is rejected for 54 processing or appropriate process adjustments are taken (e.g. blanching conditions). 
55 The objective of this study was to identify parameters which best predict the sensitivity of potatoes at 56 the start of the production process for acrylamide formation during frying French fries. To our 57 knowledge, an elaborate correlation study considering a combination of the three most obvious 58 parameters (reducing sugars, colour and acrylamide) together in French fries has not yet been 59 performed. Therefore, the correlation between reducing sugars in the raw material, the brown colouring 60 and the formation of acrylamide in this fried potato product was investigated. Furthermore, threshold 13

\section{Materials and methods}

\section{Reagents and chemicals}

All reagents and chemicals used for the acrylamide and sugar analysis were described in Mestdagh et al. (2005) and De Wilde et al. (2005) respectively.

\section{Raw material}

Potato (Solanum tuberosum L.), varieties Bintje, Fontana and Asterix (Belgium); Russet Burbank, Lady Olympia and Markies (The Netherlands), Innovator and Victoria (France); Agria (Germany); and Maris Piper (UK) were used for the study. One lot of each variety was supplied by a single producer to 74 be studied throughout storage. Additionally, all lots were approved for frying according to the industry 75 entrance control criteria (size above $50 \mathrm{~mm}$ and minimal under water weight of $380 \mathrm{~g}$ ). All tubers were 76 harvested in 2007, treated with CIPC and stored at $8^{\circ} \mathrm{C}$. Samples were taken on five different points in 77 time (January-June).

78 Fully refined palm oil (Cargill Refined Oils-Europe) was used for all the frying experiments.

\section{$79 \quad$ Preparation of French fries}


Thirty five potato tubers from each variety were peeled and cut into strips $(1 \mathrm{~cm}$ x $1 \mathrm{~cm}$ x $3 \mathrm{~cm})$ with a French fries-shaped cutter. To obtain strips with similar dimensions, parts that were in contact with the outer peel of the tuber were rejected for frying. Subsequently, only the potato strips originating from the central part of the potato were used for the experiment. These were washed with tap water for $1 \mathrm{~min}$, superficially dried on absorbent paper and divided into three groups (Figure 1). A first group, (Entrance parameters) was fried at $180^{\circ} \mathrm{C}$ for $3 \mathrm{~min}$. A second group (unblanched Final parameters) was par-fried at $180^{\circ} \mathrm{C}$ for $90 \mathrm{~s}$, frozen overnight and fried at $175^{\circ} \mathrm{C}$ for $150 \mathrm{~s}$. The third group (blanched Final parameters) included a blanching step $\left(70^{\circ} \mathrm{C}, 20 \mathrm{~min}\right.$, ratio potato/water of $1: 5$ (w:w)) prior to the parfrying step (as described for the unblanched). Frying and par-frying were conducted in a semiprofessional thermostated deep-fryer (Fritel 2505, Belgium), containing $5 \mathrm{~L}$ oil and equipped with a stirring mechanism to ensure a homogeneous temperature in the oil bath and a digital thermometer controlling the stability of the oil temperature upon frying $\left( \pm 1^{\circ} \mathrm{C}\right)$. Directly after frying, the French fries were cooled on absorbent paper and the surface colour was measured. Finally, the fries were thoroughly homogenized for subsequent acrylamide and dry matter content analysis. The repeatability of the French fries preparation method was previously assessed with a RSD of 15\% (Mestdagh et al. 2005).

\section{Acrylamide analysis}

Acrylamide was determined in homogenized potato sample by LC-MS/MS as described previously (Mestdagh et al. 2005). After aqueous extraction, using [2,3,3- $\left.\mathrm{d}_{3}\right]$ acrylamide as internal standard, the acrylamide extract was further cleaned up by solid-phase extraction. The extract was analyzed using LC-MS/MS with positive electrospray ionization.

\section{Sugar analysis}

Mono- and disaccharides in homogenized potato sample were assessed by GC-FID analysis as described earlier (De Wilde et al. 2005). Briefly, after aqueous extraction, addition of an internal standard (phenyl- $\beta$-D-glucopyranoside), clean-up and drying under nitrogen, the filtrate was derivatized and injected in a GC equipped with a flame-ionization detector. 


\section{Analysis of dry matter content}

The dry matter content was determined, based on an official AOAC method (1990). Briefly, $5 \mathrm{~g}$ of homogenized potatoes were mixed with calcinated sea sand and placed in the oven at $105^{\circ} \mathrm{C}$ until a constant weight was obtained.

\section{Colour evaluation techniques}

The colour of the French fries was measured using a Konica Minolta spectrophotometer CM-2500d (Konica Minolta, Osaka, Japan), operating in the CIE L*a*b* colour space, as described previously (Mestdagh et al. 2008a). Immediately after cooling down, the French fries were closely aligned in a plastic Petri dish. The readings were carried out in five-fold placing the instrument measuring port (8 $\mathrm{mm}$ aperture) at both sides of the dish. In such a way, the reflectance of a representative part of the surface was measured. To exclude variable cover surface conditions, the specular reflectance was included in the colour measurement. Following measurement conditions were applied: UV 100\%; standard illuminant D65 and observer angle $10^{\circ}$. The instrument was calibrated with a white calibration tile and black calibration box. Data acquisition was performed using the Spectramagic NX colour data software, version 1.52 (Osaka, Japan).

The colour was also evaluated using an Agtron process analyzer (model E15-FP, Nevada, USA), as described previously (Mestdagh et al. 2008a). Agtron values range from 0 (black) to 100 (white). The measurement was repeated three times by shaking up the fries in between each measurement. The apparatus was calibrated using a white tile (Agtron value of 100), according to manufacturer's instructions.

Finally the colour was also evaluated using an USDA/Munsell colour chart (VIVA, Den Haag, The Netherlands) which represents the method for entrance control of raw material (potatoes) currently used in French fries industry (UEITP 2007). USDA/Munsell has a seven scoring system. The system includes 000; 00 and 0 (three different degrees of very lightly coloured French fries) followed by scores $01 ; 02$; 03 and 04 (yellow to very brown French fries). All 35 fries from each variety were scored by comparing 
their colour visually with the USDA/Munsell colour chart and the average score calculated.

\section{Statistical analysis}

Pearson correlation analysis was performed using SPSS 15.0 (SPSS inc., Chicago, IL) to determine linear relationships between different parameters studied and acrylamide.

\section{Results and discussion}

Reducing sugar contents of potato tubers, colour and acrylamide formation in French fries of 10 different potato varieties

The present study included 10 varieties of potato tubers from Western Europe, representing the most frequently used in potato processing industry. Five sampling points were taken from January to June. Thus a sufficient spreading of the reducing sugar content of the raw material throughout storage could be obtained, although in practice, these varieties are not necessarily processed in the potato industry from January to June. Some varieties (Bintje, Victoria, Fonatana and Maris Piper) presented higher levels of reducing sugars on June's sampling point, while no specific trend was found throughout the initial storage period (data not shown). The reducing sugar concentrations of Asterix, Agria and Markies did not change significantly throughout the whole storage period. Due to spoilage, the varieties Innovator, Russet Burbank and Lady Olympia were not considered for the last sampling point. For these varieties the reducing sugar contents did not change either significantly from January to May.

Table 1 presents the average reducing sugar contents in raw material and acrylamide formation in French fries for the different potato varieties throughout storage. In addition, the min and max values of reducing sugar contents before blanching are represented in between brackets for each variety. These values do not necessarily correspond to the first and last sampling point for each variety. Varieties Asterix and Maris Piper presented the highest reducing sugar content in raw material (averages 0.34 -

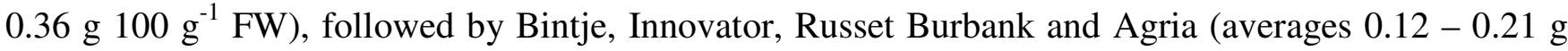
$\left.100 \mathrm{~g}^{-1} \mathrm{FW}\right)$. The lowest reducing sugar contents were observed with Lady Olympia, Victoria, Fontana 
and Markies (averages $0.03-0.06 \mathrm{~g} 100 \mathrm{~g}^{-1} \mathrm{FW}$ ). For the varieties which presented a higher reducing sugar concentration in June's sampling point, the contribution of this last point would not have influenced the variety grouping just mentioned. Since there are many factors which account for reducing sugar content in potato tubers, this hinders the comparison of this parameter for every variety with those reported in literature. Considering a relative comparison, Feltran et al. (2004) and De Wilde et al. (2006) observed, similarly as in this study, higher concentrations of reducing sugars for the variety Asterix relatively to the variety Bintje. However, both these varieties presented in this study higher reducing sugar contents comparatively to De Wilde et al. (2006), in a storage experiment carried out from the 2003 harvest. This difference illustrates the need for careful interpretation of data, with respect to possible seasonal variation or other factors influencing the level of acrylamide precursors in raw potatoes. Comparing the three varieties Bintje, Agria and Markies, results showed higher concentrations of reducing sugars for the variety Bintje compared to Agria and Markies presenting the lowest concentration of all three. This was also observed among others (Amrein et al. 2003).

Blanching is an important unit operation used in French fry processing, since it influences final product specifications, such as colour, fat content and texture (Moreira et al. 1999). In addition, during this step acrylamide precursors are leached out resulting in the reduction of acrylamide content in the final product (Mestdagh et al. 2008b). In general, potato processors increase the intensity of blanching conditions towards the end of the potato season due to senescent sweetening occurrence. These conditions needed to be kept constant during the course of the correlation study. Therefore blanching conditions applied in this study $\left(70^{\circ} \mathrm{C}\right.$ for $\left.20 \mathrm{~min}\right)$ result from a compromise of moderate blanching that would generally be applied in the beginning of the season and a stronger treatment which would be applied by the end of the season. These conditions allowed a removal of reducing sugars over $38 \%$ (Table 1). This resulted in lighter coloured French fries (data not shown) and lower levels of acrylamide (average $48-86 \%$ reduction) in the final product (Table 1). Percentages of reducing sugars removal were not directly proportional to percentages of acrylamide reduction. For example, even though the varieties 
Lady Olympia and Victoria presented the same percentage of reducing sugars removal (50\% average) after blanching, the latest presented a higher acrylamide reduction (86\% average) compared to Lady Olympia (53\% average). This observation suggests the influence on acrylamide formation of other compositional parameters which vary between cultivars besides reducing sugars.

The current quality control applied in industry at the reception of raw material potatoes (surface colour of fried unblanched potato strips evaluated with a USDA colour chart) was additionally compared to two other colour evaluation methods previously studied (Mestdagh et al. 2008a), the CIE $\mathrm{L}^{*} \mathrm{a} \mathrm{b}^{*}$ colour parameters and a process-specific Agtron analyzer. The latest is typically used in the French fries industry for on-line final product quality control. Lighter coloured French fries resulted from the reducing sugars removal during blanching treatment. This has reflected in an increase of Agtron value and a decrease of USDA and $\mathrm{a}^{*}$ values. $\mathrm{L}^{*}$ and $\mathrm{b}^{*}$ values did not vary significantly (data not shown).

Correlation study between reducing sugar content of the raw material, colour and acrylamide formation in French fries

The correlation between sugar content in the raw material, colour and acrylamide formation in French fries was investigated. Figures 2-5 demonstrate that relationships between the different parameters evaluated in this study and acrylamide are best described as linear. Different trends were observed for colour parameters and acrylamide at entrance before and after blanching, Figures 2-4 (I). Such difference was less evident when relating final parameters with acrylamide as shown by Figures 2-4 (II). Two factors may contribute for this variation. The influence of different frying conditions (one stage frying vs two stage frying and different frying temperatures) probably had an effect on acrylamide formation, and secondly the removal of other acrylamide precursors besides reducing sugars (e.g. 3-aminopropionamide) during the blanching step. On the other hand, no difference was observed between trends obtained with the parameter reducing sugars and acrylamide before and after blanching (Figure 5). Since blanching influenced the trends between the different parameters and 
acrylamide, combining the data (unblanched and blanched) for the correlation study would not be relevant. Moreover it should be realized that processed French fries are always blanched, so by keeping the data separated, the study is more representative towards the industrial practice. Therefore, Pearson correlation coefficients $(\mathrm{R})$ presented in Table 2 were determined considering acrylamide results from blanched samples only. This represents the most realistic approach to find a predictor for acrylamide content in the final product.

As mentioned in literature (Amrein et al. 2003; Becalski et al. 2004; De Wilde et al. 2005; Mestdagh et al. 2008a) for potato products, a correlation between reducing sugars and acrylamide formation was found (Figure 5 and Table 2). Similarly, no link was established between sucrose and acrylamide (De Wilde et al. 2006). A better correlation was obtained between fructose and acrylamide compared to the reducing sugar glucose. Mestdagh et al. (2008), described an excess of fructose compared to glucose stimulated acrylamide formation to a higher extent than Maillard browning.

As shown on Table 2, among $\mathrm{L}^{*} \mathrm{a}^{*} \mathrm{~b} *$ parameters, the best correlation was found between $\mathrm{a}^{*}$ and acrylamide. This was also observed by Mestdagh et al. (2008) although an exponential relationship between $\mathrm{a}^{*}$ and acrylamide was obtained. In that study higher levels of sugars and consequently higher contents of acrylamide were formed, giving rise to a different relationship. Both $\mathrm{a}^{*}$ and Agtron parameters presented a better correlation with acrylamide in the final product after blanching and a two stage frying ( $\mathrm{R}=0.80$ and $\mathrm{R}=-0.88$ respectively) compared to the short frying used for entrance control $(\mathrm{R}=0.77$ and $\mathrm{R}=-0.83$ respectively). A different trend was observed with the USDA colour evaluation method. The current entrance control (short frying test $180^{\circ} \mathrm{C}, 3$ min and USDA colour measurement), used for the prediction of other product specifications rather than acrylamide, is poorly correlated to acrylamide in the final product $(\mathrm{R}=0.74)$. This method allows only a five grade classification scale. Moreover, such classification is rather subjective since it is done by comparing visually the colour of the product with the colour chart. Analyzing reducing sugars in raw material (based on fresh weight) does not improve the correlation with the acrylamide of the final product $(\mathrm{R}=0.72)$. A better correlation is 
obtained with Agtron process analyzer after frying at $180^{\circ} \mathrm{C}$ for 3 min $(\mathrm{R}=-0.83)$. Agtron colour measurements after blanching $\left(70^{\circ} \mathrm{C}, 20 \mathrm{~min}\right)$ and a two stage frying $\left(180^{\circ} \mathrm{C}, 90 \mathrm{~s}\right.$ and $\left.175^{\circ} \mathrm{C}, 150 \mathrm{~s}\right)$ obtained the best correlation regarding acrylamide in final product $(\mathrm{R}=-0.88)$.

Based on the raw material used and the process conditions (blanching and frying) applied in this study, colour evaluation with Agtron allowed a better correlation with acrylamide compared to the reducing sugar contents. For the practical implementation in the food industry, using a colour parameter as an acrylamide predictor in raw material is more practical than analyzing sugar contents. However, results indicate that the current quality control of raw material potatoes, can be improved towards acrylamide prediction by changing colour evaluation method from USDA colour chart to the Agtron process analyzer.

The correlation study combined with a specific acrylamide level in French fries could provide an extra preventive tool in their food safety management system, based on threshold values (THV) of the evaluated parameters best related to acrylamide. Such approach applied as a quality control measurement for raw potatoes at the reception point could prevent the acrylamide problem during further production.

\section{Evaluation of threshold values}

The correlation study discussed above revealed the parameter best correlated to acrylamide formation in French fries was obtained with Agtron measurements. Reducing sugars are also a relevant parameter since they are chemically known to be the limiting precursor for acrylamide formation in potatoes. On the other hand USDA is the current quality control tool used for the approval or rejection of incoming potatoes in French fry industry. Consequently, these relevant parameters were considered for the determination of THV presented in Table 3. The calculation of such values was based on the linear relationships obtained between the different parameters with the acrylamide formed in the final product and considering a hypothetical acrylamide limit of $500 \mu \mathrm{g} \mathrm{kg}^{-1}$. Relationships between colour 
parameters and acrylamide are represented by the broken line on Figures 2-3 (I - entrance parameters and II - final parameters) and relationships between reducing sugars and acrylamide on Figure 6 (solid line - entrance parameter and broken line - final parameter). The hypothetical acrylamide limit was selected based on the most recent signal value $\left(530 \mu \mathrm{g} \mathrm{kg}^{-1}\right)$ established for French fries /chips by the German minimization concept for acrylamide. The signal value is defined as the lowest level of the $10 \%$ of products of each commodity group that have the highest acrylamide content (Göbel and Kliemant 2007). The denominations "acceptable" (acrylamide $<500 \mu \mathrm{g} \mathrm{kg}^{-1}$ ) and "rejectable" (acrylamide $>500$ $\mu \mathrm{g} \mathrm{kg}^{-1}$ ) will be adopted throughout the discussion of the results. In practice, incoming potatoes that would be classified as "rejectable" (within a certain limitation) would not be necessarily rejected but rather subjected to a more rigorous blanching treatment. This would allow further sugar removal thus rendering the French fries less prone to acrylamide formation and suit final product specifications (acrylamide $<500 \mu \mathrm{g} \mathrm{kg}^{-1}$ ). The application of the THV to the samples analyzed in this study resulted in a preliminary analysis of sample categorization (Table 3). Samples which are categorized as "acceptable" and have acrylamide $>500 \mu \mathrm{g} \mathrm{kg}^{-1}$ (third column of Table 3) could represent a nonconformity. On the other hand, samples which are categorized as "rejectable" and present acrylamide levels $<500 \mu \mathrm{g} \mathrm{kg}^{-1}$ (fifth column on Table 3) would have been in conformity (in respect to the acrylamide limit). Both scenarios are undesirable, however the first scenario could compromise final consumer safety, and the second scenario represents unnecessary "extra-precautions for industry" (e.g., extra blanching) which translates in unnecessary costs.

The highest percentage of rejected samples (33\%) was obtained with the application of the USDA THV after a short frying test (USDA <2.1). Worth noticing is the fact that $50 \%$ of those rejected samples correspond to samples "unnecessarily rejected". Similarly, the application of the Agtron THV ( $>57)$ after the short frying test presented a high percentage of samples rejected with acrylamide $<500$ $\mu \mathrm{g} \mathrm{kg}^{-1}(45 \%)$. This value was reduced to $17 \%$ with the application of the THV for reducing sugars in

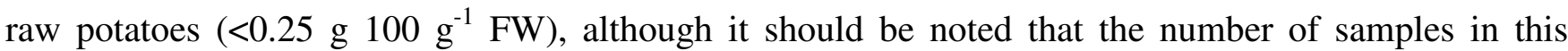


category was too small to draw final conclusions.

On the other hand, considering the application THV for the final parameters (after blanching and fried in two stages) different trends were obtained. The application of a THV for USDA $<1.6$ remains a poor tool for predicting acrylamide in the final product, meaning that $56 \%$ of rejected samples had

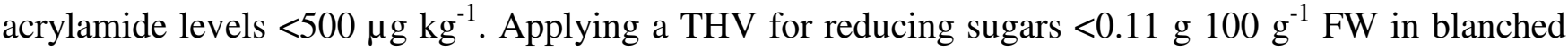
potatoes increased the percentage of rejected samples with acrylamide $<500 \mu \mathrm{g} \mathrm{kg}^{-1}$ from $17 \%$ to $25 \%$. In contrast, the application of a THV for Agtron $>61.0$ showed major improvements. This application resulted in a reduction of "unnecessarily rejected" samples to only $22 \%$ leading to the highest percentage of correctly categorized samples (93\%). Although this investigation was done on a lab scale, and in addition a sensory evaluation of the final product was not considered, the THV obtained for Agtron in the final product fits within the range of limits of consumer acceptability. Generally considered, the final product colour is too bright at Agtron values above 75 and too dark below 55 (Mestdagh et al. 2008a).

When selecting a quality control parameter for raw material potatoes at the reception for acrylamide mitigation purposes, an important aspect to consider when evaluating the THV application is the final consumer safety (percentage of accepted samples with acrylamide $>500 \mu \mathrm{g} \mathrm{kg}^{-1}$ ). These percentages ranged from 3 to $12 \%$ for the parameters presented on Table 3. The lowest percentage (3\%) was obtained with the application of the THV for Agtron final (after blanching and fried in two stages). The parameter USDA final (after blanching and fried in two stages) allowed the highest percentage of accepted samples with acrylamide $>500 \mu \mathrm{g} \mathrm{kg}^{-1}(12 \%)$.

It should be kept into account that the THV for quality control tools mentioned in this paper are directly related to the hypothetical $500 \mu \mathrm{g} \mathrm{kg}^{-1}$ acrylamide level and thus are limited to the blanching and frying conditions used in this study. As such, the THV mentioned should not be considered as absolute. Because of the impact of final frying on acrylamide formation, an approach in which more appropriate selection criteria of the raw material combined with informative advices given to consumers 
and catering may contribute to safer French fries.

The preliminary data obtained in this study and the threshold values concept presented, demonstrate that from a food safety point of view, a more optimal quality control could be adopted for incoming potatoes in order to guarantee reduced acrylamide contents in the final product. Agtron measurement after blanching and a two stage frying, or, measuring reducing sugars of the incoming product could provide a preventive tool for French fry industry to evaluate the acrylamide problem at the start of the production. It should be emphasized that implementing these alternatives of quality control in for instance a food safety management system on company level, would require the input of supplementary resources, having an impact on total production cost and cost-effectiveness of these possible mitigation tools.

Since acrylamide formation is dependent on the surface area of the potato product and on the process conditions applied, the extrapolation of results for other type of potato products with different surface-to volume ratios, such as potato crisps, should not be considered.

Moreover, due to the multiple pre and post-harvest factors that may impact the final amounts of the precursors in the raw material to acrylamide in French fries, this study should be repeated/validated throughout different seasons. Work is currently been carried out for the 2008 harvest season.
Abbreviations used
DM, dry matter; FW, fresh weight; AA, acrylamide; RS, reducing sugars; THV, threshold value; USDA, United States Department of Agriculture.

\section{Acknowledgments}

This research was financed by Flanders' FOOD, EPPA and Belgapom. Frédéric Mestdagh is a post- 
doctoral researcher supported by the research Foundation-Flanders (FWO). The support provided by

PinguinLutosa Foods (Belgium) for the necessary practical arrangements was greatly appreciated. The authors thank the steering committee of EPPA and Dr. Liesbeth Jacxsens for fruitful discussions during editing of the manuscript.

\section{References}

Amrein TM, Bachmann S, Noti A, Biedermann M, Barbosa MF, Biedermann-Brem S, Grob K, Keiser A, Realini P, Escher F and Amadò R. 2003. Potential of acrylamide formation, sugars, and free asparagine in potatoes: A comparison of cultivars and farming systems. J Agric Food Chem. 51: 55565560.

AOAC . 1990. Association of Official Analytical Chemists. Moisture in animal feed. Official Methods of Analysis, 930.15, AOAC, Gaithersburg, MD.

Becalski A, Lau BPY, Lewis D and Seaman SW. 2003. Acrylamide in foods: occurrence, sources, and modeling. J Agric Food Chem. 51: 802-808.

Becalski A, Lau BPY, Lewis D, Seaman SW, Hayward S, Sahagian M, Ramesh M and Leclerc Y. 2004. Acrylamide in French fries: influence of free amino acids and sugars. J Agric Food Chem. 52: 38013806.

Biedermann M, Noti A, Beidermann-Brem S, Mozzetti V and Grob K. 2002. Experiments on acrylamide formation and possibilities to decrease the potential of acrylamide formation in potatoes. Mitteilungen aus Lebensmitteluntersuchung und Hygiene. 93: 668-687.

Blenkinsop RW, Copp LJ, Yada RY and Marangoni AG. 2002. Changes in compositional parameters of tubers of potato (Solanum tuberosum) during low-temperature storage and their relationship to chip processing quality. J Agric Food Chem. 50: 4545-4553.

CIAA. 2006. The CIAA acrylamide "toolbox" (http://www.ciaa.be/documents/brochures/CIAA_Acrylamide_Toolbox_Oct2006.pdf)

De Wilde T, De Meulenaer B, Mestdagh F, Govaert Y, Ooghe W, Fraselle S, Demeulemeester K, Van Peteghem C, Calus A, Degroodt JM and Verhé R. 2006. Selection criteria for potato tubers to minimize acrylamide formation during frying. J Agric Food Chem. 54: 2199-2205.

De Wilde T, De Meulenaer B, Mestdagh F, Govaert Y, Vandeburie S, Ooghe W, Fraselle S, Demeulemeester K, Van Peteghem C, Calus A, Degroodt JM and Verhé R. 2005. Influence of storage practices on acrylamide formation during potato frying. J Agric Food Chem. 53: 6550-6557.

Feltran JC, Lemos LB and Vieites RL. 2004. Technological quality and utilization of potato tubers. 
Scientia Agricola. 61: 598-603.

Foot RJ, Haase NU, Grob K and Gonde P. 2007. Acrylamide in fried and roasted potato products: A review on progress in mitigation. Food Additives and Contaminants. 24: 37-46.

Göbel A and Kliemant A. 2007. The German minimization concept for acrylamide. Food Additives and Contaminants. 24: 82-90.

IARC. 1994. Acrylamide. International Agency for Research on Cancer, Lyon, France

Mestdagh F, De Wilde T, Castelein P, Németh O, Van Peteghem C and De Meulenaer B. 2008a. Impact of the reducing sugars on the relationship between acrylamide and Maillard browning in French fries. European Food Research and Technology. 227: 69-76.

Mestdagh F, De Wilde T, Fraselle S, Govaert Y, Ooghe W, Degroodt JM, Verhe R, Van Peteghem C and De Meulenaer B. 2008b. Optimization of the blanching process to reduce acrylamide in fried potatoes. Lwt-Food Science and Technology. 41: 1648-1654.

Mestdagh F, Lachat C, Baert K, Moons E, Kolsteren P, Van Peteghem C and De Meulenaer B. 2007. Importance of a canteen lunch on the dietary intake of acrylamide. Molecular Nutrition \& Food Research. 51: 509-516.

Mestdagh FJ, De Meulenaer B, Van Poucke C, Detavernier C, Cromphout C and Van Peteghem C. 2005. Influence of oil type on the amounts of acrylamide generated in a model system and in French fries. J Agric Food Chem. 53: 6170-6174.

Moreira RG, Castell-Perez M and Barrufet MA (1999) Deep-fat frying: fundamentals and applications. Aspen Publishers, Gaithersburg, MD, 350 pp. pp.

Mottram DS, Wedzicha BL and Dodson AT. 2002. Acrylamide is formed in the Maillard reaction. Nature. 419: 448-449.

Stadler RH, Blank I, Varga N, Robert F, Hau J, Guy PA, Robert MC and Riediker S. 2002. Acrylamide from Maillard reaction products. Nature. 419: 449-450.

Tareke E, Rydberg P, Karlsson P, Eriksson S and Törnqvist M. 2002. Analysis of acrylamide, a carcinogen formed in heated foodstuffs. J Agric Food Chem. 50: 4998-5006.

UEITP. 2007. Entrance control for raw material (potatoes) currently used in the French fries industry (www.ueitp.org) 
2

\section{Figure captions}

Figure 1. Experimental setup used for the correlation study between reducing sugars in raw material, colour and acrylamide formation in French fries.

Figure 2. Relation between USDA (I - after short frying test; II - after two stage frying) and acrylamide content (determined after two stage frying) in unblanched (filled square, solid line) and blanched (open square, broken line) French fries.

Figure 3. Relation between Agtron (I - after short frying test; II - after two stage frying) and acrylamide content (determined after two stage frying) in unblanched (filled square, solid line) and blanched (open square, broken line) French fries.

Figure 4. Relation between $\mathrm{a}^{*}$ colour (I - after short frying test; II - after two stage frying) and acrylamide content (determined after two stage frying) in unblanched (filled square, solid line) and blanched (open square, broken line) French fries.

Figure 5. Relation between reducing sugars content in raw potatoes and acrylamide content in French fries (after two stage frying) without blanching (filled square, solid line) and after blanching (open square, broken line).

Figure 6. Relation between reducing sugars content in raw potatoes without blanching (filled square, 
411 solid line) and after blanching (open square, broken line) with acrylamide content in French fries (after

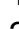
3412 blanching and two stage frying).

4

5

6

7

8

9

10

11

12

13

14

15

16

17

18

19

20

21

22

23

24

25

26

27

28

29

30

31

32

33

34

35

36

37

38

39

40

41

42

43

44

45

46

47

48

49

50

51

52

53

54

55

56

57

58

59

60 


\section{Table captions}

2

3 4 6415 7

8 9 10 11 $12^{41}$ 13 14 $15^{418}$ 16
Table 1. Average dry matter (DM), reducing sugars content (RS) and acrylamide (AA) formation in French fries before and after blanching treatment for different potato varieties throughout storage. RS contents between brackets represent min and max values.

Table 2. Pearson correlation coefficients $(\mathrm{R})$ between respectively entrance parameters (reducing sugars-raw material; colour-1 stage frying) and final parameters (reducing sugars-blanched raw material; colour-after blanching and two stage frying) and acrylamide (after blanching and two stage frying) levels in French fries.

Table 3. Threshold values determination for entrance parameters (reducing sugars-raw material; colourafter one stage frying) and final parameters (reducing sugars-blanched raw material; colour-after blanching and two stage frying), based on a hypothetical acrylamide limit of $500 \mu \mathrm{g} \mathrm{kg}^{-1}$ and their application to samples of the present study regarding the acrylamide formation in French fries. 


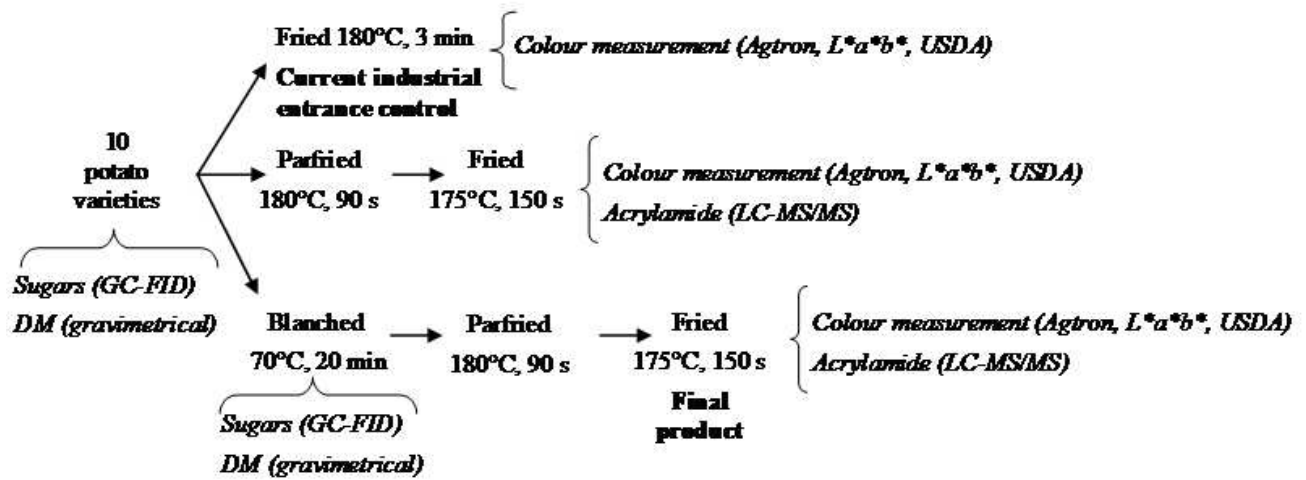

Figure 1. Experimental setup used for the correlation study between reducing sugars in raw material, colour and acrylamide formation in French fries. $213 \times 84 \mathrm{~mm}(96 \times 96 \mathrm{DPI})$ 
Figure 2. Relation between USDA (I - after short frying test; II - after two stage frying) and acrylamide content (determined after two stage frying) in unblanched (filled square, solid line) and blanched (open square, broken line) French fries. $200 \times 94 \mathrm{~mm}(96 \times 96 \mathrm{DPI})$ 
Figure 3. Relation between Agtron (I - after short frying test; II - after two stage frying) and acrylamide content (determined after two stage frying) in unblanched (filled square, solid line) and blanched (open square, broken line) French fries. $196 \times 95 \mathrm{~mm}(96 \times 96 \mathrm{DPI})$ 
Figure 4. Relation between a* colour (I - after short frying test; II - after two stage frying) and acrylamide content (determined after two stage frying) in unblanched (filled square, solid line) and blanched (open square, broken line) French fries. $200 \times 91 \mathrm{~mm}(96 \times 96 \mathrm{DPI})$ 


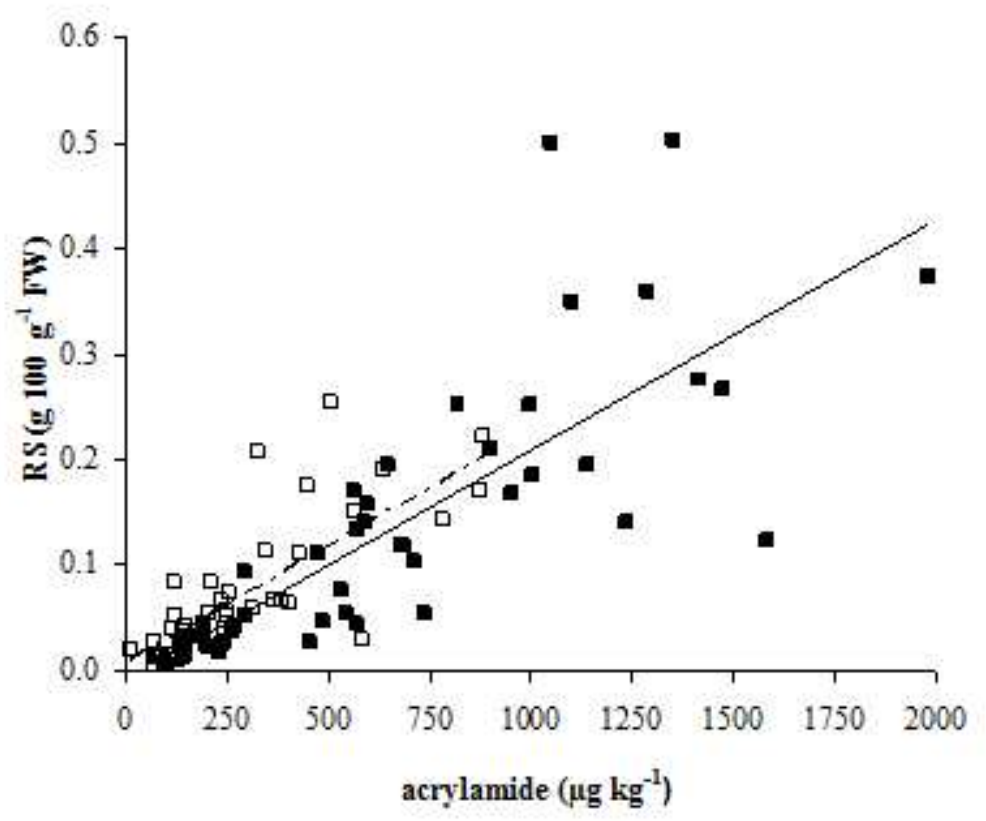

Figure 5. Relation between reducing sugars content in raw potatoes and acrylamide content in French fries (after two stage frying) without blanching (filled square, solid line) and after blanching (open square, broken line). $102 \times 89 \mathrm{~mm}(96 \times 96 \mathrm{DPI})$ 
Figure 6. Relation between reducing sugars content in raw potatoes without blanching (filled square, solid line) and after blanching (open square, broken line) with acrylamide content in French fries (after blanching and two stage frying).

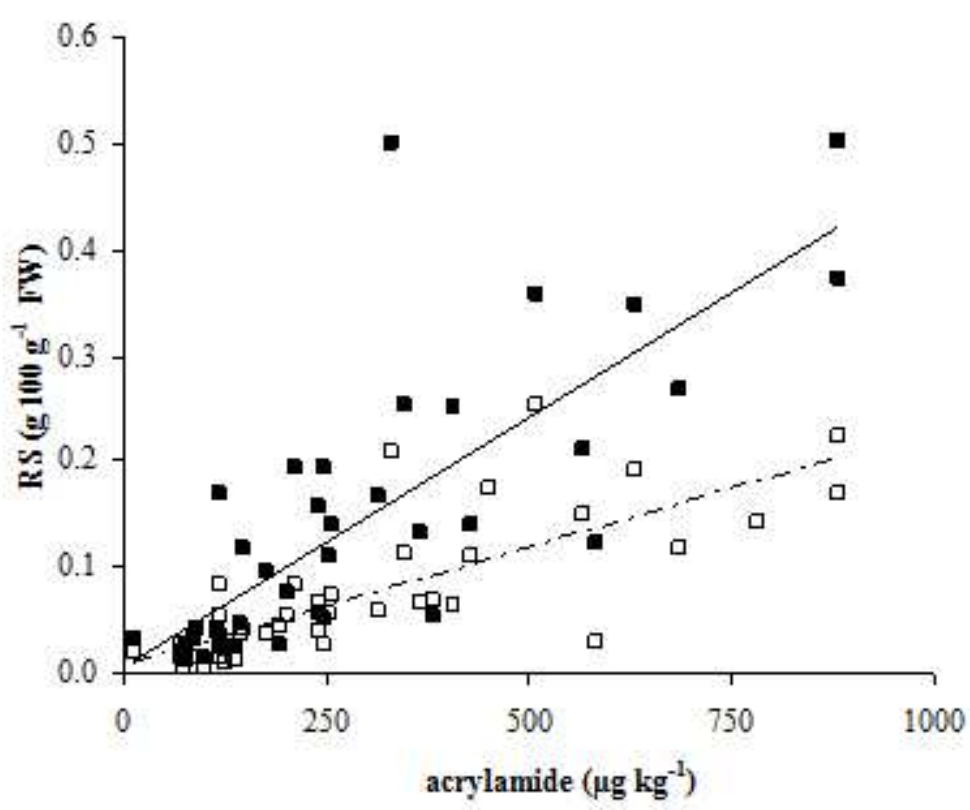
$102 \times 91 \mathrm{~mm}(96 \times 96 \mathrm{DPI})$ 
Table 1. Average dry matter (DM), reducing sugars content (RS) and acrylamide (AA) formation in French fries before and after blanching treatment for different potato varieties throughout storage. RS contents between brackets represent min and max values. $135 \times 91 \mathrm{~mm}(150 \times 150 \mathrm{DPI})$ 
Table 2. Pearson correlation coefficients $(R)$ between respectively entrance parameters (reducing sugars-raw material; colour-one stage frying) and final parameters (reducing sugars-blanched raw material; colour-after blanching and two stage frying) and acrylamide (after blanching and two stage frying) levels in French fries. $176 \times 173 \mathrm{~mm}(96 \times 96 \mathrm{DPI})$

\begin{tabular}{|c|c|c|}
\hline & Parameter & $\mathrm{R}$ \\
\hline \multirow{9}{*}{$\underset{\text { 式 }}{\stackrel{g}{\stackrel{E}{E}}}$} & USDA & $0.74^{3}$ \\
\hline & Agtron & $-0.83^{3}$ \\
\hline & $L^{*}$ & $-0.63^{3}$ \\
\hline & $a^{*}$ & $0.77^{\mathrm{a}}$ \\
\hline & $b^{*}$ & $-0.46^{b}$ \\
\hline & $\mathrm{RS}\left(\mathrm{g} 100 \mathrm{~g}^{-1} \mathrm{FW}\right)$ & $0.72^{\mathrm{a}}$ \\
\hline & Fructose $\left(\mathrm{g} 100^{-1} \mathrm{FW}\right)$ & $0.75^{\mathrm{a}}$ \\
\hline & 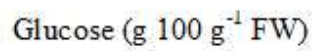 & $0.70^{\mathrm{a}}$ \\
\hline & Sucrose $\left(\mathrm{g} 100 \mathrm{~g}^{-1} \mathrm{FW}\right)$ & $0.00^{b}$ \\
\hline \multirow{9}{*}{$\underset{\Xi}{\text { 至 }}$} & USDA & $0.68^{2}$ \\
\hline & Agtron & $-0.88^{3}$ \\
\hline & $L^{*}$ & $-0.59^{3}$ \\
\hline & $a^{*}$ & $0.80^{\mathrm{a}}$ \\
\hline & $b^{*}$ & $-0.22^{b}$ \\
\hline & $R S\left(g 100 g^{-1} F W\right)$ & $0.78^{\mathrm{a}}$ \\
\hline & 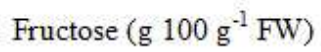 & $0.83^{\mathrm{a}}$ \\
\hline & 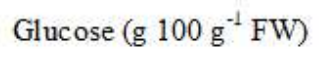 & $0.65^{3}$ \\
\hline & Sucrose $\left(\mathrm{g}^{\left.100 \mathrm{~g}^{-1} \mathrm{FW}\right)}\right.$ & $0.29^{b}$ \\
\hline
\end{tabular}


Table 3. Threshold values determination for entrance parameters (reducing sugars-raw material; colour- after one stage frying) and final parameters (reducing sugars-blanched raw material; colourafter blanching and two stage frying), based on a hypothetical acrylamide limit of $500 \mu \mathrm{gg}-1$ and their application to samples of the present study regarding the acrylamide formation in French fries. $182 \times 83 \mathrm{~mm}(96 \times 96 \mathrm{DPI})$ 